\title{
When Does Abusive Supervision Affect Job Performance Positively?
}

\author{
Fuda Li $\mathbb{D}^{\prime}$, Bangzhe Tan', Li Zhou', Huaqian Huang² \\ 'Business School, Hunan Normal University, Changsha, People's Republic of China; ${ }^{2}$ College of Tourism and Sport Health, Hezhou University, Hezhou, \\ People's Republic of China \\ Correspondence: Li Zhou, Business School, Hunan Normal University, Changsha, Hunan Province, 4I0000, People's Republic of China, \\ Tel +86 I327245600I, Email lizhou@hunnu.edu.cn
}

Purpose: By combining the Job Demand-Resources model with Arnold's action sequence as the overall logic, this study explores the connections between abusive supervision and job performance.

Participants and Methods: This study employed two-point surveys, with 474 valid responses, to reduce the risk of common method bias. On this basis, confirmatory factor analysis was used to test the reliability and validity of data, and Smart-PLS was used to test the hypotheses.

Results: Our findings suggest that abusive supervision has a significant positive impact on job performance. Furthermore, challenge stressors and innovative work behavior partially mediate the relationship between abusive supervision and job performance, and these two factors also form a chain mediating effect. Leader-member exchange moderates the relationship between abusive supervision and job performance, as higher levels of leader-member exchange are associated with a stronger positive effect of abusive supervision on job performance.

Practical Implications: This study provides insights to managers about the link between abusive supervision and employee performance. In addition, it recommends that leaders at all levels adopt abusive supervision when they can properly consider a specific employee's perception of the reasons for their behavior and must consider the previous inclusion in the manager's circle of members, as well as the link between the challenging pressures they face and innovative work behavior to deal with such management behaviors.

Originality: Most research on abusive management has focused on its negative effects on employee performance. This study, by contrast, explores whether there are positive impacts from abusive management and when such positive effects will occur.

Keywords: abusive supervision, challenge stressors, innovative work behavior, leader-member exchange, job performance

\section{Introduction}

Abusive supervision refers to persistent verbal or non-verbal hostility shown by supervisors towards their subordinates. ${ }^{1}$ The literature indicates that the specific characteristics of abusive supervision include ridicule, public criticism, loud outbursts, rudeness, indifference to subordinates, coercion, and use of contemptuous language. ${ }^{1,2}$ Studies have shown that abusive supervision is subjective, continuous, hostile, and non-physical. ${ }^{1}$ Research also suggests that abusive supervision often has a negative impact on subordinate employees in areas such as sharing knowledge, ${ }^{3}$ work attitude, ${ }^{4}$ job performance, ${ }^{5}$ and job behaviors, ${ }^{6}$ and there are even reductions in the company's performance. ${ }^{1}$

Abusive supervision has been recognized as negative leadership behavior. However, the influence of cultural background is rarely considered in relation to abusive supervision and employee performance. For example, in China, the corporate culture of forced overtime in internet companies is significant and widespread, and employees often experience abusive supervision from their superiors. Indeed, in 2019, there was even an incident where programmers in the "996 ICU" (a work schedule that expects employees to work from 9 am to 9 pm, six days a week without overtime pay) rebelled against forced overtime. Nevertheless, Chinese internet companies like Alibaba Group, Tencent, and ByteDance have flourished in recent years. This seems to contradict the mainstream research on abusive supervision, as there might 
not be a negative correlation between abusive supervision and employee performance. Rather, the effects of abusive supervision on job performance might vary in different cultural backgrounds.

Some researchers have examined the positive effects of abusive supervision. Lee et al argued that appropriate abusive supervision depletes employee resources but it enhances other resources, such as creativity, ${ }^{7,8}$ and this might improve team task performance in the long term. Conversely, in Western cultures, employees may be more sensitive to human rights and perceive abusive supervision as a violation of these rights, enhancing the negative impact of this type of supervision. ${ }^{4}$ Therefore, Asian culture may have a moderating role in distinguishing between the negative and positive effects of abusive supervision. ${ }^{9}$

However, it is important to understand how abusive supervision can be used effectively to ensure it has positive rather than negative effects on employee performance. The intermediate variables in this process and any boundary conditions this too shall pass should be investigated. Indeed, researchers have not found direct links between these issues. In fact, in the Chinese context of management having high power and being paternalistic, leaders may consider being harsh towards their subordinates to motivate them to work hard. ${ }^{9}$ However, pure fear does not provide a comprehensive explanation for the complex relationship between abusive supervision and job performance. There must be other influencing factors. Therefore, it is of great importance to help employees improve their job performance by ensuring that subordinates correctly perceive the value of abusive supervision and transform this pressure into motivation.

The Job Demand-Resources model ${ }^{10}$ states that positive outcomes in the workplace are related to the appropriate balance of job requirements and resources, and that high job requirements and high job resources enable employees to invest greater energy in innovation ${ }^{11}$ to increase work performance. ${ }^{12,13}$ In addition, the central assumption of the JD-R model is that job strain develops - irrespective of the type of job or occupation — when job demands are high and when job resources are limited. ${ }^{10}$ Thus, from the JD-R model, we can identify two variables that have some association with job performance: challenging stress and innovative work behavior. Challenge stressors are job demands that employees perceive as rewarding work experiences, such as workload, time pressure, job duties, and job complexity, ${ }^{14}$ and these are mainly reflected in the initiative of the individual employees. Innovative work behavior refers to the generation, dissemination, and implementation of new ideas in the workplace, ${ }^{15}$ arising mainly from the employees' knowledge or skill base.

The American psychologist Arnold, on the subject of emotion and motivation, suggested a sequence of "cognitiveevaluative-emotional-need-thoughts-act". ${ }^{16}$ Based on this, abusive supervision may first affect subordinates' perception of their superiors' behaviors, which is followed by social comparison and self-evaluation. Second, the effects of abusive supervision are also a reflection of the individual's emotional responses, which, to a certain extent, also represent the individual's emotions. In this context, challenge stressors and innovative work behavior represent the "need" and "thoughts" parts of the action sequence respectively. Finally, the individual's choice of behavior represents the "act". JD-R suggests that high job demands can be emotionally debilitating, while the lack of job resources can have a decisive impact on individual motivation and behavior, ultimately leading to separation from the job and a decrease in selfefficacy. ${ }^{17}$ As a result, individuals tend to be more motivated to innovate and change so they can alleviate the emotional drain of abusive management and ensure consistency between cognition and behavior. ${ }^{18}$ Therefore, this paper uses a chain-mediated pathway based on the combination of the Job Demand-Resources model and Arnold's action sequence to investigate the mechanisms of the relationship between abusive supervision and employee job performance.

An individual's behavior can be significantly influenced by their interpersonal relationships. ${ }^{19}$ Indeed, studies suggest that employees receive more resources when the quality of the leader-member exchange is higher. ${ }^{20}$ Having access to more resources enables employees to manage the challenge stressors of the job better, and it increases the likelihood that the challenge will drive innovation. In terms of the JD-R model, employees who belong to the same social circle as their leader may have access to both sufficient work resources and psychosocial support to cope with the demands of the job. ${ }^{21}$ This pattern is strongly linked to the circle culture in Chinese companies today, where it is easier to gain trust and support when there are closer personal relationships between employees and leaders. However, in Chinese companies, there is often paternalistic leadership situation. Leaders often perceive themselves as the parents or elders of their subordinates and, thus, criticize or even insult their subordinates' mistakes to motivate them to work hard. This is in line with the traditional Chinese family education rule of "spare the rod and spoil the child" ${ }^{22,23}$ Researchers have suggested that to 
study the impact of abusive management, the quality of the leader-member exchange also should be considered and its marginal effects included in the study. ${ }^{24,25}$

In summary, this paper explores a mediation and moderation model of the effect of abusive supervision on job performance through challenge stressors and innovative work behavior. This approach is based on the Job DemandResources model in conjunction with Arnold's action sequence. This study also analyses the influence of leader-member exchange as a boundary condition of the model. Using survey data from a Chinese context, this investigation provides important insights into the mechanisms underpinning the effect of abusive supervision on job performance, and it offers new ideas and practical guidance regarding the effective use of abusive supervision to improve employee job performance.

\section{Theory and Hypotheses}

\section{Abusive Supervision and Job Performance}

Since Tepper introduced the concept of abusive supervision in $2000,{ }^{26}$ research on this common negative leadership behavior has continued. Initially, examinations of the concept of abusive supervision focused on its essential features rather than its specific outcomes. ${ }^{27}$ Conceptually, researchers have identified three main characteristics of abusive supervision: the subjective nature of its evaluation, the continuity of the behavior, and the purposeful nature of the abuse. $^{2}$

In terms of the purpose of abusive supervision, researchers tend to support the perspective that leaders use it to motivate their subordinates to improve their performance or to indicate that mistakes will not be tolerated so they are not repeated. This suggests that the purpose of abusive supervision is at least positive, as opposed to the purpose of workplace aggression. ${ }^{2}$ Many researchers have linked abusive supervision to negative outcomes such as reduced innovation, disruption of the organizational climate, and impediments to business development. ${ }^{1,4}$ However, the effect of abusive supervision on employee performance remains controversial. ${ }^{9,28}$

The reasons for abusive supervision can also change its impact on employee performance. Individuals make attributions about the cause of abusive supervision, and abusive supervision is more likely to generate anger, further transgressive behavior, and less organizational citizenship behavior when individuals think abusive supervision is being used to cause harm. However, when subordinates perceive that the motivation for abusive supervision is to improve performance, they are more likely to have feelings of guilt leading to less deviant behavior and more involvement in organizational citizenship. ${ }^{18}$ Especially in the Chinese context, the attitude of managers towards their employees is commonly paternalistic. Therefore, the purpose of abusive supervision is predominantly to motivate subordinates to work hard and to reduce mistakes in their work rather than to be abusive. ${ }^{5,28}$ Therefore, we propose the following hypothesis:

Hypothesis 1: Abusive supervision is positively associated with employee performance in Chinese organizational contexts.

\section{The Mediating Role of Challenge Stressors}

Abusive supervision is a continuous and complex process based on a sequence of cognitive-evaluative-emotional components. These represent three basic psychological aspects of the individual, and they create challenging stressors.

There are various aspects to abusive supervision. First, individuals become psychologically distressed and they become more willing to change to achieve their work goals. The aim is to avoid further psychological distress from such supervision and to reduce the likelihood of being abused. ${ }^{29}$ Second, abusive supervision increases perceived workplace stress. ${ }^{1}$ However, unlike workplace aggression, abusive supervision is not seen as unavoidable, so the individual may try to transform the stress of abusive supervision into creative motivation. ${ }^{18}$ Finally, by managing the pressure of challenging work, the individual's performance and competencies can be enhanced. Then, the leader becomes better able to perceive the individual's contributions, and the individual may feel positive about the previous motivation and encouragement. The result is the development of a positive leader-member exchange relationship. ${ }^{30}$ In summary, abusive supervision can give individuals challenging stressors that motivate them to work hard and that stimulate their desire for recognition. Based on this analysis, we propose the following hypothesis: 
Hypothesis 2: Abusive supervision is positively associated with challenge stressors.

Stress at work is ubiquitous, but it can be differentiated based on whether it is beneficial to the individuals. Stressors that present a challenge and stimulate achievement, positive emotions, and good outcomes are known as challenge stressors. In contrast, stressors that hinder effective performance and the achievement of work objectives are known as hindrance stressors. ${ }^{14,31}$ Resources conservation theory states that when individuals are aware of changes in resources this significantly influences their attitudes and behavior. ${ }^{32}$ Specifically, when individuals perceive a certain level of stress at work, which indicates available resources have changed, this helps them concentrate on their work. They endeavor to acquire to acquire new resources to reduce the net loss of resources, and this enhances job performance. ${ }^{31}$ There is a wealth of research on the relationship between challenge stressors and job performance, and there is consensus that challenge stressors motivate individuals to work hard, thus improving their job performance. ${ }^{14,31,33}$ Based on this analysis, we propose the following hypotheses:

Hypothesis 3: Challenge stressors are positively associated with employee performance.

Hypothesis 4: Challenge stressors positively mediate the relationship between abusive supervision and job performance, such that abusive supervision enhances the challenge stressors on employees, leading to improved job performance.

\section{The Mediating Role of Innovative Work Behavior}

With innovative work behavior, employees generate, disseminate, and implement new ideas in their work. ${ }^{15}$ Abusive supervision is a destructive leadership behavior that represents a negative situational factor, and the impact of such negative factors on employees' attitudes and behaviors is of high research value. This is because they stimulate more responses from individuals than positive situational factors, meaning they have a greater impact on employees' attitudes and behaviors. ${ }^{34}$ Most researchers believe that abusive supervision hurts employees' innovative work behavior. $^{35}$

As innovation causes high levels of risk and uncertainty, it requires both leadership support and a supportive working environment. ${ }^{36}$ When there are negative situational factors like abusive supervision in the workplace, supervisors may be indifferent to the ideas of their subordinates. Moreover, the dissemination or implementation of new ideas may even be perceived as a challenge to the authority of the supervisors. ${ }^{37}$ However, by focusing on employee outcomes after they engage in innovative behavior rather than their reasons for these behaviors, researchers have overlooked several key issues. ${ }^{6}$ Indeed, abusive supervision is not a singular event. It is ongoing, long-term, emotional, and psychologically nonbenevolent treatment of subordinates. ${ }^{1}$ Therefore, if a subordinate wishes to change from being managed with constant abuse, they must show a breakthrough in their work. ${ }^{29}$ This explains why employees who are abusively managed still wish to be innovative in their work. Based on this analysis, we propose the following hypothesis:

Hypothesis 5: Abusive supervision is positively associated with innovative work behavior.

However, new ideas alone cannot entirely alter the evaluation and perception of subordinates by superiors, so they must also produce beneficial results. Studies have shown that employees' innovative work behavior is important for improving their job performance. ${ }^{38}$ This means that individuals can change the way their leaders perceive them by using innovative work behavior to produce positive outcomes. In that case, abusive supervision may encourage employees to be innovative in the workplace, and employee job performance must improve for leaders to recognize the role of individual innovation and provide more leadership support. Based on this analysis, we propose the following hypotheses:

Hypothesis 6: Innovative work behavior is positively associated with employee performance.

Hypothesis 7: Innovative work behavior mediates the relationship between abusive supervision and job performance, such that abusive supervision reinforces innovative work behavior, improving employee performance. 


\section{The Joint Mediating Role of Challenge Stressors and Innovative Work Behavior}

Researchers have divided stress into challenge stressors and hindrance stressors, and these two types of stressors have different effects on innovative work behavior. There is consensus regarding the negative impact of hindrance stressors on innovation, ${ }^{39}$ and most researchers are positive about the specific relationship between challenge stressors and employee innovation. They argue that challenge stressors may motivate employees to overcome challenges and work hard, and this is conducive to innovation. ${ }^{40}$ However, challenge stressors do not always lead to positive outcomes. They can also deplete the cognitive resources of employees, thus diminishing their positive effect on innovation. ${ }^{41,42}$

Studies have shown that employees are most motivated and most engaged in the process of innovation when their access to resources and their job requirements both are at a high level. ${ }^{43}$ This is because, according to the JD-R model, high levels of job resources counteract the resource-depleting effects of challenge stressors. This enhances the motivational power of challenge stressors and reinforces their positive effects. ${ }^{11,43}$ Based on this above analysis, we propose the following hypothesis:

Hypothesis 8: Challenge stressors are positively associated with innovative work behavior.

Furthermore, the emotion-motivation-action sequence suggests that there are important interactions between individuals' perceptions and actions and that these factors have reciprocal influences on each other. ${ }^{16}$ Therefore, when individuals form a perception of an event, they actively evaluate the situation to identify any gaps in their abilities. This process generates emotions, so the cognitive-evaluative-emotional sequence creates specific cognitions, evaluations, emotions, needs, thoughts, and behaviors. ${ }^{44}$ This suggests that the cognitive-evaluative-emotional sequence may influence individual behavior both by satisfying certain psychological needs and through a process of continuous reflection.

Based on this logic, challenge stressors enhance basic work needs by motivating employees to work harder and belong to the need unit in the action sequence. Moreover, innovation requires continuous reflection, so employees innovate by thinking about how to change their current situation. This belongs to the thinking unit in the action sequence. Specifically, when an individual experiences persistent hostile behavior, either verbal or non-verbal, from their superiors, and especially when others are not treated in the same way, this can lead the person to think that they are less skilled than others, thus causing emotional distress. To alleviate such distress or prevent it from happening again, the individual may reflect on and change their previous behaviors. ${ }^{18}$

Taken together, abusive supervision, with its cognitive-evaluative-emotional aspects, may increase challenge stressors (need) on employees, encouraging them to think and formulate new ideas (innovative work behavior), and this may ultimately lead to changes in their behavioral outcomes (job performance). Based on the above analysis, we propose the following hypothesis:

Hypothesis 9: Challenge stressors and innovative work behavior together mediate the relationship between abusive supervision and job performance, such that abusive supervision enhances job performance by increasing individuals' challenge stressors and, thus, their innovative work behavior.

\section{The Moderating Role of Leader-Member Exchange}

Leader-member exchange refers to the interpersonal relationship between leaders and subordinates that is established through their work. This creates two types of groups based on relationship affinity: the inner circle and the outer circle. ${ }^{45}$ As leaders have limited resources of time and energy, they may not be particularly concerned about each of their subordinates. As a result, the subordinates who are recognized by the leader, known as insiders, receive extra care, attention, and social resources from the leader, including job information and career guidance. In contrast, the individuals who receive fewer resources from the leader are referred to as outsiders. ${ }^{20}$ In the traditional Chinese interpersonal environment, being an insider means having a good social relationship with the leader and other individuals close to them, and this optimizes the atmosphere in the workplace. ${ }^{6}$ The individual can even become the leader's assistant, supporting the leader to advance their own career. Moreover, insiders can satisfy their own needs and use their insider 
status to gain access to the intentions and beliefs of senior leaders. ${ }^{21}$ This enhances their own decisions to create valuable work and, ultimately, drive the organization's competitiveness.

In contrast, outsiders with low levels of leader-member exchange are easy targets for abusive supervision. However, subordinates who enjoy high levels of leader- member exchange also can be abused by their leaders. ${ }^{46}$ Indeed, leadership is often paternalistic in the Chinese context. The managers in the company act as parents and educate and manage their employees as if they were children, but they also care for them in their lives and work, and they endeavor to support their growth and development through criticism and care. ${ }^{22,23}$ From an attribution perspective, employees who see that their supervisors are abusively managing them for positive reasons may experience a sense of guilt that motivates them to work harder. ${ }^{18}$ Therefore, the contributions of abusive supervision to job performance may be stronger when there is a higher level of leader-member exchange. Based on this analysis, we propose the following hypothesis:

Hypothesis 10: Leader-member exchange moderates the positive relationship between abusive supervision and job performance.

This paper provides an in-depth analysis of the interactions between the core variables of abusive supervision, challenge stressors, innovative work behavior, and job performance. This analysis is based on the Job DemandResources model, according to which increased job requirements and job resources relate to increased challenge stressors and innovative work behavior. Two separate mediation pathways were created to explore how abusive supervision may improve the performance of subordinates by increasing both job requirements and job resources. Concurrently, the Arnold action sequence was used as a guide to construct the "abusive supervision-challenge stressors-innovative work behavior-job performance" (AS-CS-IW-JP) chain of mediation pathways. It did this by using abusive supervision as the cognitive- evaluative-emotional unit, challenge stressors as a need unit, and innovative work behavior as a reflection unit. The research model is presented in Figure 1.

\section{Materials and Methods}

\section{Measures}

The following actions were taken to ensure that the measurement tools had good reliability and validity. First, we used international journals to identify reliable scales that were published by authoritative bodies and validated in the Chinese context. As the scales were developed in English-speaking contexts, we used a translation-back-translation process to develop the scales. For this, we invited two Ph.D. students from the United States and the United Kingdom to translate the English scales into Chinese. Following this, two Ph.D. students in the field of management were invited to translate the scales back into English. Finally, two professors of English were invited to compare the three versions of the scales and suggest any changes needed to avoid linguistic ambiguities.

Abusive supervision is considered a negative form of leadership. Therefore, because of the sensitive and personal nature of the questions, the questionnaire was administered in an informal setting using anonymous self-assessment. Furthermore, to ensure the rigor of the survey process and the accuracy of the questionnaire, we invited 30 masters of business administration students from a university to conduct a simulation survey. The simulation showed a $100 \%$ valid return rate for the questionnaire and the students gave valuable feedback. In addition, we used a 5-point Likert scale to measure all responses $(1=$ totally disagree, $5=$ totally agree $)$. The sources of the scales are as follows:

\section{Abusive Supervision}

Aryee et al's contextually appropriate 10-item scale, based on the Tepper scale, ${ }^{1,47}$ was used to assess employees' feelings towards abusive supervision. Items included "My supervisor often says I am not competent" and "My supervisor often mentions my past mistakes or failures."

\section{Challenge Stressors}

Six items from the challenge stressors dimension of the Challenge-Hindrance Stressors Scale developed by Cavanaugh (2000) and Zhang (2014) were used. Challenge stressors relate to workload, time pressure, task complexity, and responsibility. One item was "The amount of work I have to complete within the time limit". ${ }^{14,42}$ 


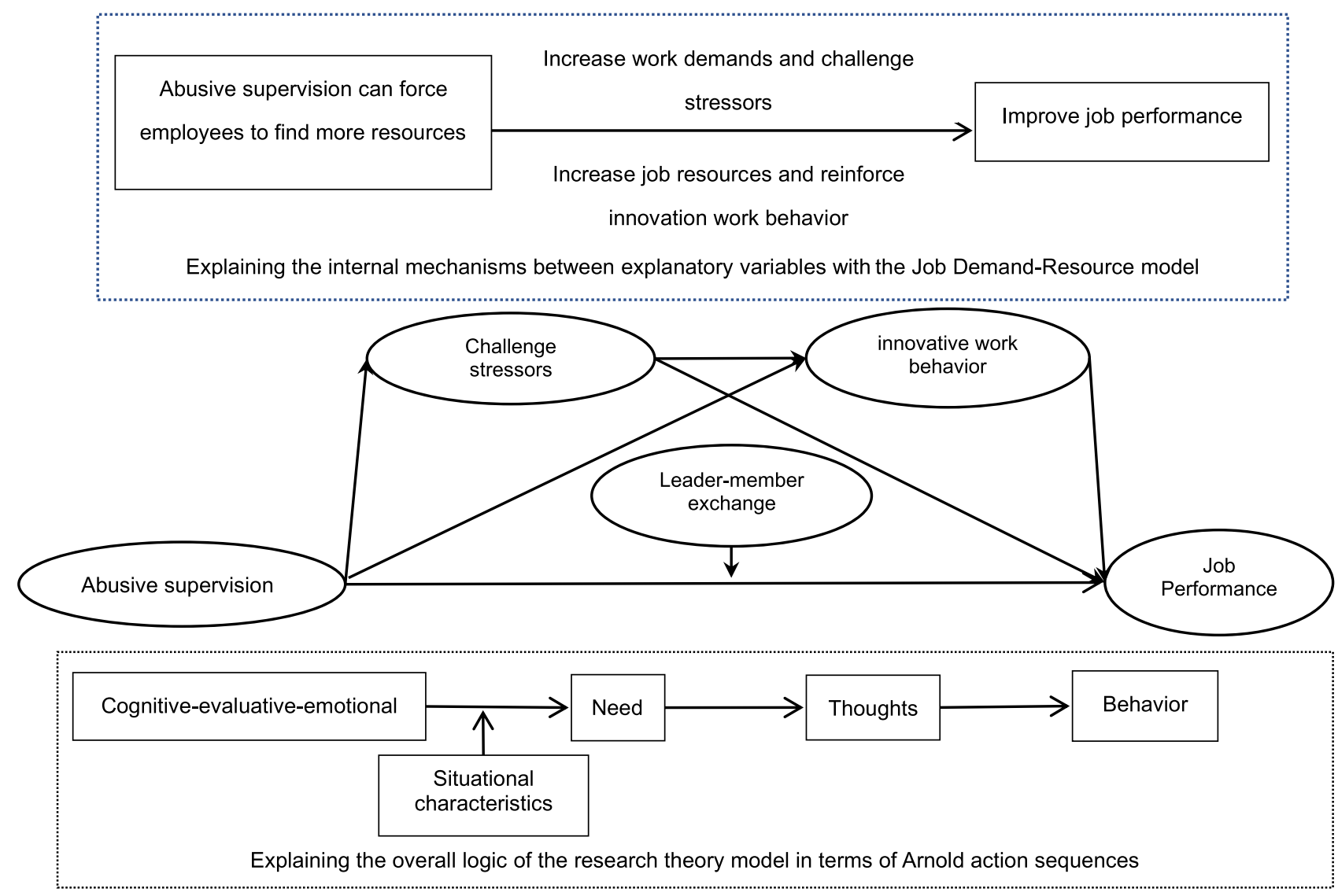

Figure I Conceptual Model for this Research.

\section{Innovative Work Behavior}

Scott and Bruce's (1994) five-item scale was used to measure employees' innovative work behavior. ${ }^{15}$ Items included "I come up with new ideas" and "I search for new ideas on technology, processes, techniques, and products."

\section{Leader-Member Exchange}

Six statements were based on the scale of leader-member exchange developed by Graen and Uhl-Bien (1995). They included "I have enough confidence in my leader to champion and defend his or her decisions if he or she is not present."

\section{Job Performance}

The job performance scale developed by Chen et al was used. ${ }^{48}$ There were four statements, including "I have made a significant contribution to the overall performance of our unit," and "I am one of the best performers in our unit."

\section{Samples and Procedures}

The participants came from several of China's most developed cities, including the first-tier cities of Beijing, Shanghai, Guangzhou, and Shenzhen, and the second-tier cities of Changsha and Foshan. In line with the purpose of this study and the meaning of the variables, industries with high-performance pressure, high prevalence of management behaviors such as heckling and ridicule, and high employee turnover were selected. They included finance, internet, insurance, and real estate transaction. Moreover, the companies had all been established for more than 5 years, and they had 200 or more employees. The online form of informed consent was received from all individuals involved in the study. The subjects willingly and voluntarily took part in the research. The Institutional Review Board of the Hunan Normal University approved the study design and data collection. All procedures involving human participants were conducted in accordance with the ethical standards of the institutional research committee, the ethical standards of the APA, and the 1964 Helsinki Declaration and its later amendments or comparable ethical standards. 
To avoid the serious issue of homologation error, ${ }^{49}$ including different Likert scale formats, we adopted two time points for collecting the questionnaires. We reminded the employees that the questionnaires were anonymous, and we included some interference in the items. At time point 1, data were collected regarding the respondents' basic personal information, contact details, perceptions of abusive supervision, leader-member exchange; 30 days later, at time point 2, information regarding challenge stressors, innovative work behavior, and job performance was collected again from the same group respondent.

The first step of the investigation process was to use social networking to find a research facilitator from the target company, provide them with brief training, invite them to participate in the entire pre-research process, and ensure they understood the procedures, contents, and considerations of the study. In the second step, blank questionnaires were placed in an envelope and given to the facilitator for direct distribution to the participants in a later stage of the study. The facilitator was asked to collect the questionnaire, seal it in the envelope, and number the envelope as soon as the subjects had given their responses. In the third step, each company office was asked to distribute one copy of the questionnaire to one participant to avoid any instances of cross-referencing or communication between subjects that may affect the results of the questionnaire. In the fourth step, before the formal survey, the facilitator informed the subjects that the questionnaire would be completed anonymously in an informal setting and the results would be used for academic research only. In the fifth step, the facilitator waited near the subject while they answered the questionnaire. If the subject had any questions, they could ask the facilitator at any time. In the sixth step, all the participants were given a bottle of shampoo prepared by the research team, and the facilitator was also given US $\$ 5$.

Initially, 550 questionnaires were distributed at the first time point, and 521 questionnaires were returned (a 94.7\% response rate). Questionnaires with incomplete responses, obvious non-compliance, or irregular responses were excluded, leaving 481 responses. Then, the last 4 digits of the cell phone numbers filled in by the employees were matched with the employee address book provided by the company, so 474 valid responses remained, yielding an overall questionnaire efficiency of $86.18 \%$.

As shown in Table 1, 41.77\% of the respondents were male and $58.23 \%$ were female. In terms of age, $46.84 \%$ were 18-29 years old, $48.10 \%$ were $30-39$ years old, $1.27 \%$ were $40-49$ years old, and $3.80 \%$ were 50 years old and above. In

Table I Background Characteristics of Participants ( $N=474)$

\begin{tabular}{|c|c|c|c|}
\hline \multicolumn{2}{|l|}{ Characteristics } & \multirow{2}{*}{$\begin{array}{c}\text { Frequency } \\
198\end{array}$} & \multirow{2}{*}{$\begin{array}{c}\text { Percentage } \\
41.77\end{array}$} \\
\hline Sex & Male & & \\
\hline & Female & 276 & 58.23 \\
\hline \multirow[t]{4}{*}{ Age category } & $18-29$ & 222 & 46.84 \\
\hline & $30-39$ & 228 & 48.10 \\
\hline & $40-49$ & 6 & 1.27 \\
\hline & $50+$ & 18 & 3.80 \\
\hline \multirow[t]{4}{*}{ Highest education completed } & Junior high school and below & 6 & 1.27 \\
\hline & High school & 18 & 3.80 \\
\hline & Bachelor's degrees & 366 & 77.22 \\
\hline & Master's degrees or above & 84 & 17.72 \\
\hline \multirow[t]{5}{*}{ Working age } & $<1$ & 72 & 15.19 \\
\hline & $1-3$ & 138 & 29.11 \\
\hline & $3-5$ & 78 & 16.46 \\
\hline & $5-10$ & 114 & 24.05 \\
\hline & $10+$ & 72 & 15.19 \\
\hline
\end{tabular}


terms of education, $1.27 \%$ completed junior high School and below, 3.80\% completed high school, $77.22 \%$ had bachelor's degrees, and $17.72 \%$ had master's degrees or above. In terms of working age, $15.19 \%$ were in their first year, $29.11 \%$ were there between 1 and 2 years, $16.46 \%$ were there between 3 and 4 years, 24.05\% were there between 5 and 9 years, 15.19 were 10 years or more.

\section{Results}

Partial least squares (PLS) was used because it is suitable for identifying causal relationships between the construct variables, and it can deal with both model constructs and measurement items. ${ }^{50}$ In addition, PLS is suitable for handling relationships between variables in anomalous data distributions because of its relaxed requirements for normality and randomness of variables. ${ }^{51}$

\section{Measurement Model}

We used structural equation modeling (SEM) with Smart-PLS and SPSS software to investigate our research hypotheses. The results of reliability and validity testing with Smart-PLS are presented in Table 2. Table 2 shows that all composite reliabilities of the constructs were greater than 0.7 , indicating there was good internal consistency of the measures for each construct. Table 2 also shows that all average variance extracted (AVE) values were above $0.5,{ }^{52}$ and the square root of each construct's AVE was greater than the correlation of the construct with other latent variables. This indicated the convergent and discriminant validity of the constructs., Table 3 shows that each item loads more highly on its own construct than on other constructs, indicating good discriminant validity.

\section{Structural Model and Hypothesis Testing}

We used bootstrapping (bootstrapping samples $\mathrm{N}=5000$ ) to calculate $t$-statistics and standard errors. ${ }^{53}$ Figure 2 presents the results of the structural model without the moderator variable of leader-member exchange, and Figure 3 shows the results of the structural model with the moderator variable for the relationship between abusive supervision and job performance. As shown in Figures 2 and 3, all paths were positive and significant $(p<0.01)$. The structural model (Figure 2), omitting the moderation effect, explained $50.0 \%$ of the variance in challenge stressors, $53.1 \%$ of the variance in innovative work behavior, and $68.0 \%$ of the variance in job performance. In contrast, the moderated model with leader-member exchange (Figure 3) accounted for $70.7 \%$ of the variance in job performance. Therefore, based on the structural model with the moderator variable, we ran hypothesis testing. The results of hypothesis testing (Table 4) supported all 10 hypotheses.

There were high correlations between certain constructs; for example, the correlation between abusive supervision and innovative work behavior was 0.689 . This may lead to collinearity, which can magnify or obscure the relationships between constructs. ${ }^{54}$ Henseler suggested that the variance inflation factor (VIF) can be used to test for collinearity, and

Table 2 Construct Reliability and Validity

\begin{tabular}{|c|c|c|c|c|c|c|c|c|}
\hline \multirow[t]{2}{*}{ Construct } & \multirow[t]{2}{*}{$\alpha$} & \multirow[t]{2}{*}{ CR } & \multirow[t]{2}{*}{ AVE } & \multicolumn{5}{|c|}{ Discriminant Validity (Fornell-Larcker Criterion) } \\
\hline & & & & ( & (2) & (3) & (4) & (5) \\
\hline (I) AS & 0.966 & 0.971 & 0.767 & 0.876 & & & & \\
\hline (2) CS & 0.875 & 0.906 & 0.618 & 0.707 & 0.786 & & & \\
\hline (3) IWB & 0.888 & 0.918 & 0.692 & 0.689 & 0.655 & 0.832 & & \\
\hline (4) JP & 0.909 & 0.936 & 0.785 & 0.703 & 0.656 & 0.790 & 0.886 & \\
\hline (5) LMX & 0.930 & 0.945 & 0.740 & -0.467 & $-0.34 I$ & -0.349 & -0.478 & 0.860 \\
\hline
\end{tabular}

Notes: Diagonal bold is the square root of average variance extracted (AVE). For adequate discriminant validity, the diagonal elements should be greater than the corresponding off-diagonal elements.

Abbreviations: $\alpha$, Cronbach's Alpha; CR, composite reliability; AVE, average variance extracted; AS, abusive supervision; CS, challenge stressors; IWB, innovative work behavior; JB, job performance; LMX, Leader-Member Exchange. 
Table 3 Factor Loadings and Cross Loadings for the Measurement Model

\begin{tabular}{|c|c|c|c|c|c|}
\hline Items & AS & CS & IWB & JP & LMX \\
\hline ASI & 0.858 & 0.558 & 0.485 & 0.508 & -0.334 \\
\hline AS2 & 0.898 & 0.562 & 0.519 & 0.535 & -0.358 \\
\hline AS3 & 0.87 I & 0.545 & 0.483 & 0.500 & -0.333 \\
\hline AS4 & 0.815 & 0.586 & 0.538 & 0.562 & -0.346 \\
\hline AS5 & 0.899 & 0.680 & 0.644 & 0.655 & -0.440 \\
\hline AS6 & 0.899 & 0.692 & 0.669 & 0.704 & -0.387 \\
\hline AS7 & 0.868 & 0.584 & 0.586 & 0.595 & -0.457 \\
\hline AS8 & 0.910 & 0.628 & 0.639 & 0.649 & -0.477 \\
\hline AS9 & $0.86 I$ & 0.650 & 0.615 & 0.602 & -0.460 \\
\hline ASIO & 0.876 & 0.659 & $0.76 \mathrm{I}$ & 0.762 & -0.458 \\
\hline CSI & 0.605 & 0.810 & 0.527 & 0.526 & -0.302 \\
\hline CS2 & 0.522 & 0.750 & 0.478 & 0.477 & -0.239 \\
\hline CS3 & 0.645 & 0.870 & 0.563 & 0.552 & -0.348 \\
\hline CS4 & 0.613 & 0.776 & 0.525 & 0.512 & -0.336 \\
\hline CS5 & 0.472 & 0.730 & 0.425 & 0.443 & -0.224 \\
\hline CS6 & 0.455 & 0.772 & 0.558 & 0.574 & -0.142 \\
\hline IWBI & 0.500 & 0.508 & 0.786 & 0.584 & -0.248 \\
\hline IWB2 & 0.598 & 0.566 & 0.857 & 0.674 & -0.302 \\
\hline IWB3 & 0.588 & 0.551 & 0.840 & 0.650 & -0.273 \\
\hline IWB4 & 0.610 & $0.58 \mathrm{I}$ & 0.842 & 0.693 & -0.343 \\
\hline IWB5 & 0.560 & 0.515 & $0.83 I$ & 0.676 & -0.280 \\
\hline JPI & 0.695 & 0.607 & 0.732 & 0.916 & -0.578 \\
\hline JP2 & 0.605 & 0.574 & 0.713 & 0.882 & -0.365 \\
\hline JP3 & 0.584 & 0.534 & $0.68 I$ & 0.880 & -0.350 \\
\hline JP4 & 0.601 & 0.607 & 0.670 & 0.866 & -0.383 \\
\hline LMXI & -0.433 & -0.304 & -0.288 & -0.432 & $0.85 I$ \\
\hline LMX2 & -0.424 & -0.290 & -0.305 & -0.427 & 0.873 \\
\hline LMX3 & -0.378 & -0.270 & -0.297 & -0.391 & 0.863 \\
\hline LMX4 & -0.383 & -0.286 & -0.306 & -0.408 & 0.850 \\
\hline LMX5 & -0.386 & -0.302 & -0.281 & -0.405 & 0.878 \\
\hline LMX6 & -0.402 & -0.308 & -0.325 & -0.404 & 0.846 \\
\hline
\end{tabular}

Notes: Boldface numbers are loadings for indicators to their own construct; other numbers are cross-loadings.

Abbreviations: AS, abusive supervision; CS, challenge stressors; IWB, innovative work behavior; JB, job performance; LMX, Leader-Member Exchange. 


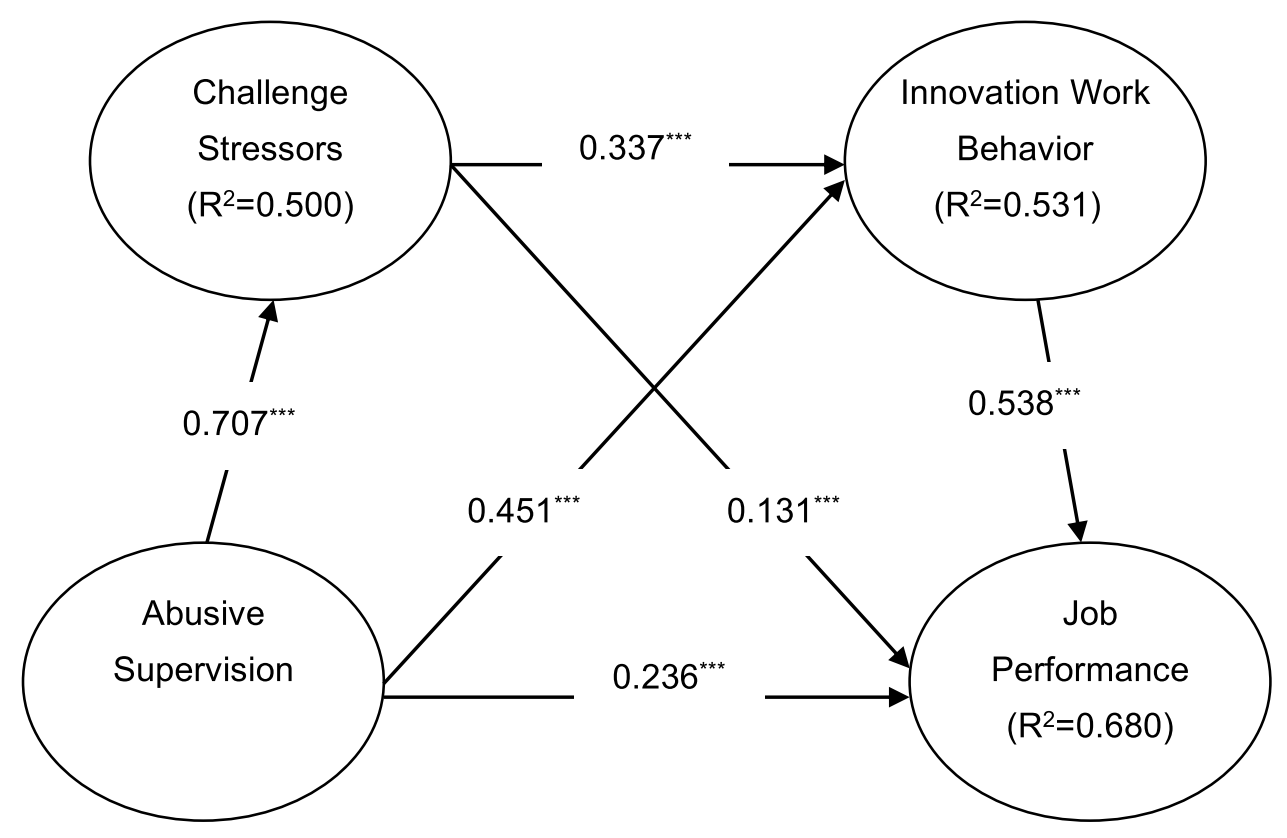

Figure 2 Structural Model without Moderator Variable.

Notes: $* * * P<0.001 . R^{2}=S S R / S S T=1-S S E / S S T ; S S T=S S R+S S E$.

Abbreviations: SST, total sum of squares; SSR, regression sum of squares; SSE, error sum of squares.

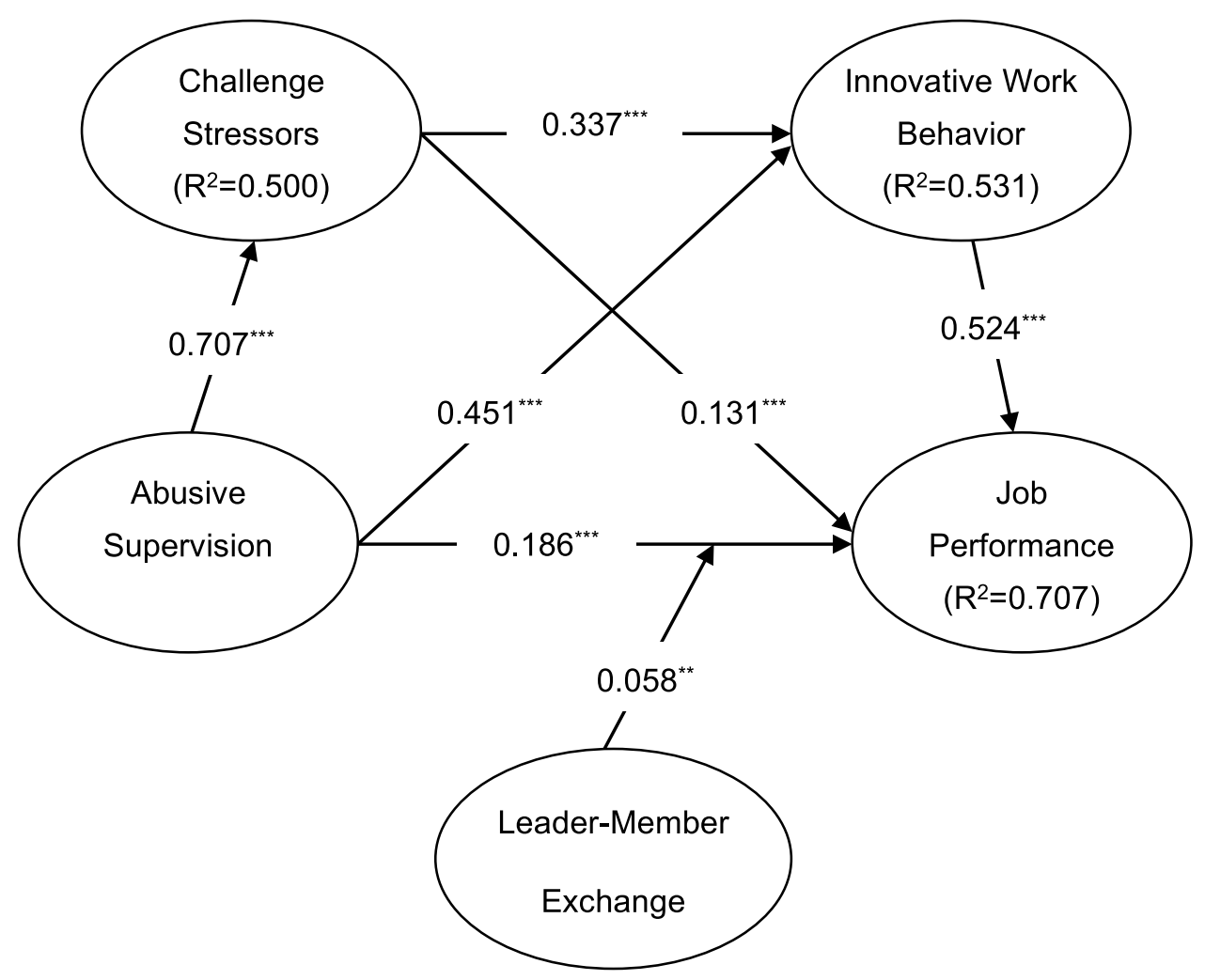

Figure 3 Structural Model with Moderator Variable.

Notes: $* * P<0.01, * * * P<0.001 . R^{2}=S S R / S S T=I-S S E / S S T ; S S T=S S R+S S E$.

Abbreviations: SST, total sum of squares; SSR, regression sum of squares; SSE, error sum of squares. 
Table 4 Results of Hypotheses Testing

\begin{tabular}{|c|c|c|c|c|c|}
\hline Hypotheses & $\boldsymbol{\beta}$ & STDEV & t Value & Bias-Corrected BCl (95\%) & Result \\
\hline $\mathrm{HI}: \mathrm{AS} \rightarrow \mathrm{JP}$ & $0.186^{* * *}$ & 0.040 & 4.687 & {$[0.108,0.265]$} & Supported \\
\hline $\mathrm{H} 2: \mathrm{AS} \rightarrow \mathrm{CS}$ & $0.707^{* * *}$ & 0.021 & 33.048 & {$[0.664,0.784]$} & Supported \\
\hline $\mathrm{H} 3: \mathrm{CS} \rightarrow \mathrm{JP}$ & $0.131^{* * *}$ & 0.039 & 3.370 & {$[0.057,0.210]$} & Supported \\
\hline $\mathrm{H} 4: \mathrm{AS} \rightarrow \mathrm{CS} \rightarrow \mathrm{JP}$ & $0.093 * * *$ & 0.028 & 3.329 & {$[0.039,0.147]$} & Supported \\
\hline $\mathrm{H} 5: \mathrm{AS} \rightarrow \mathrm{IWB}$ & $0.45 I^{* * *}$ & 0.044 & 10.323 & {$[0.365,0.535]$} & Supported \\
\hline H6: IWB $\rightarrow J P$ & $0.524 * * *$ & 0.040 & 13.125 & {$[0.445,0.600]$} & Supported \\
\hline $\mathrm{H} 7: \mathrm{AS} \rightarrow \mathrm{IWB} \rightarrow \mathrm{JP}$ & $0.236 * * *$ & 0.027 & 8.640 & {$[0.183,0.291]$} & Supported \\
\hline $\mathrm{H} 8: \mathrm{CS} \rightarrow \mathrm{IWB}$ & $0.337^{* * *}$ & 0.049 & 6.939 & {$[0.238,0.431]$} & Supported \\
\hline $\mathrm{H} 9: \mathrm{AS} \rightarrow \mathrm{CS} \rightarrow \mathrm{IWB} \rightarrow \mathrm{JP}$ & $0.125 * * *$ & 0.022 & 5.645 & {$[0.085,0.171]$} & Supported \\
\hline HIO: AS*LMX $\rightarrow J P$ & $0.058 * *$ & 0.021 & 2.833 & {$[0.018,0.099]$} & Supported \\
\hline
\end{tabular}

Notes: $* * P<0.01$, $* * * P<0.001$; bootstrapping samples= 5000; AS*LMX is interaction item.

Abbreviations: $\beta$, direct effect, or mediated effect, or moderated effect; Bias-corrected $\mathrm{BCl}$, Bias-corrected bootstrapping confidence intervals; $\mathrm{AS}$, abusive supervision; CS, challenge stressors; IWB, innovative work behavior; JB, job performance; LMX, Leader-Member Exchange.

a VIF greater than 10 indicates harmful collinearity. ${ }^{55}$ Table 5 presents the VIFs for the constructs in the model with the moderator variable. These VIFs did not exceed 3, showing that collinearity did not influence the results.

\section{Discussion}

Much research into human resources has been focused on how to transform management behaviors that are negative for individuals into positive behaviors that can improve their job performance. This paper explored the mechanisms underpinning the effects of abusive supervision on job performance, using the Job Demand-Resources model and Arnold's action sequence. There were several key findings. First, abusive supervision has a significant positive impact on job performance. In terms of its purpose, superior managers may use abusive supervision to motivate subordinates to work hard and reduce mistakes in their work. This is different from abusive behavior itself. Individuals who are aware of the supervisor's purpose may feel a sense of guilt, adopt various methods to reduce this guilt, and thus improve their job performance.

Second, there is positive mediation from both challenge stressors and innovative work behavior on the relationship between abusive supervision and job performance. (1) Abusive supervision has a positive impact on job performance by reinforcing challenge stressors. Individuals perceive that abusive supervision increases challenge stressors, and they

Table 5 The Variable Inflation Factor (VIF) for Constructs

\begin{tabular}{|l|c|c|c|}
\hline Construct & CS & IWB & JP \\
\hline AS & 1.000 & 2.000 & 2.799 \\
\hline CS & & 2.000 & 2.246 \\
\hline IWB & & & 2.140 \\
\hline LMX & & & 1.295 \\
\hline AS*LMX & & & 1.120 \\
\hline
\end{tabular}

Note: AS*LMX is interaction item.

Abbreviations: AS, abusive supervision; CS, challenge stressors; IWB, innovative work behavior; JB, job performance; LMX, Leader-Member Exchange. 
may transform the resulting stress into motivation to avoid continuous abusive supervision. This would lead them to work harder to improve their performance. (2) Abusive supervision also positively affects job performance by reinforcing innovative work behavior. As mentioned above, to avoid the psychological pain of continuous abusive supervision, employees may use active learning and innovative behavior to change the current supervision situation, and this ultimately improves their job performance. (3) Together, challenge stressors and innovative work behavior mediate the connection between abusive supervision and job performance. Abusive supervision stimulates the psychological pain arising from cognitive and behavioral inconsistencies, and it reinforces individuals' challenge stressors. The challenge stressors provide motivation for innovative work behavior, which in turn translates to improved job performance.

Third, positive leader-member exchange relationships strengthen the connection between abusive supervision and job performance. China is a humane society in which subordinates belong to the same circle as their superiors. Even if subordinates are abused by their superiors, they may feel that their superiors are helping and educating them. Therefore, they may continue to work harder to repay the care of their leaders with practical actions.

\section{Theoretical Implications}

The Job Demand-Resources model helps to explain the positive effect of abusive supervision on individual job performance. Most previous research on abusive supervision has considered it a negative leadership behavior, thus ignoring the purpose of abusive supervision by leaders and underestimating the ability of individuals to adapt at work. This has resulted in insufficient research on abusive supervision as a facilitator of individual job performance.,

The framework of this study was based on the first half of Arnold's action sequence. It was applied to the psychological purpose of abusive supervision in the traditional Chinese interpersonal environment. Based on this, individuals experience the whole cognitive-evaluative-emotional sequence in response to abusive supervision. In addition, this study used the Job Demand-Resources model to analyze the internal mechanisms underpinning the ability of abusive supervision to enhance individual job performance through inconsistencies between cognition and behavior. For example, the individual feels guilty so they work harder to counteract that feeling.

Understanding the internal mechanism of this relationship has deepened the understanding of the positive effects of abusive supervision in the workplace. Furthermore, the findings of this paper support the work of Zhang et al, who reported that the relationship between abusive supervision and job performance was indeed controversial, and abusive supervision had inconsistent roles across different team culture. ${ }^{5,9}$ In addition, our study deepens previous findings by investigating the Chinese cultural context, in which abusive supervision has positive effects on individual job performance, even when there are high levels of leader-member exchange.

Arnold's action sequence of cognitive-evaluative-emotional-need-thoughts-act helps to explain the logical relationships between the variables in this study. This reveals the mechanisms underpinning the relationship between abusive supervision and job performance more systematically and profoundly. To a certain extent, this also extends the application of Arnold's action sequence from psychology to human resource management. In the past, studies have mainly focused on the first or second half of the action sequence, and they have analyzed single meanings of the variables. ${ }^{18,56}$ This paper has verified the discrete mediating roles of challenge stressors and innovative work behavior on the relationship between abusive supervision and job performance, using the Job Demand-Resources model. Moreover, the validity of the cognitive- evaluative-emotional-need-thoughts-act action sequence to explain the chain of mediating factors between abusive supervision and job performance also has been confirmed.

Referring to the need unit in terms of challenge stressors and the thinking unit in terms of innovative work behavior, different meanings of the same variable can be identified from different theories. Through this, a rational integration of the discrete mechanisms and the entire model can provide clear insight into how abusive supervision improves job performance.

In summary, this paper enriches the research regarding the mechanisms underpinning the effect of abusive supervision on enhancing job performance, and it uses Arnold's action sequence as a new theoretical basis for exploring the consequential variables related to abusive supervision. It also broadens the scope of its application to the field of human resources. 


\section{Practical Implications}

Organizations should recognize the positive effects of abusive supervision and use it to increase motivation through various methods. First, creating a Chinese paternalistic team culture and enhancing the level of the leader-member exchange may represent an interpersonal method for motivating staff. Second, it may be beneficial to organize regular mental health counseling or training to guide staff to understand the purpose of abusive supervision (negative management behavior) and to reduce individual emotional resistance.

Leaders also should address the extent and purpose of abusive supervision and actively guide their employees to use it to improve their job performance. Primarily, leaders should examine their own levels of abusive supervision and the resilience of their team members, use varying levels of abusive supervision with different individuals, and endeavor to treat team members equally. Furthermore, they should reduce the levels of abusive supervision or publicly recognize and reward team members once they have achieved certain goals or levels of job performance. Second, to enhance job performance, leaders should provide their employees with the necessary opportunities and motivation to be innovative and encourage them to change their current situations through innovation.

As far as possible, individuals should also perceive abusive supervision as positive leadership behavior with goodwill and confront and manage the stresses that exist in the workplace. Indeed, actively participating in mental health training may be helpful for employees to understand the intent of certain leadership management behaviors better and to recognize their own resilience under stress. In addition, actively communicating and learning from other team members to acquire new knowledge, skills, and other resources can provide a solid foundation for improving job performance.

\section{Limitations and Recommendations for Future Research}

Although this paper has reported important findings regarding the relationship between abusive supervision and job performance, there are still some limitations that should be noted. First, all variables were measured only once, but abusive supervision is an ongoing negative leadership behavior that may be influenced by the respondent's mood and other factors. People have different levels of self-esteem and tolerance for abusive supervision, so these survey findings may be affected by bias. Second, we have discussed the relationship between abusive supervision and job performance only from a linear perspective, and there is already research that shows that the relationship between abusive supervision and job performance is not always linear. Therefore, adding multiple survey time points may also lead to further understanding of this subject. Third, the survey respondents were only from companies with a Chinese cultural background, and the paternalistic leadership culture may lead to increased tolerance of abusive supervision by employees. Therefore, the cultural backgrounds of the respondents should be expanded in future research.

\section{Conclusion}

By combining the Job Demand-Resources model with Arnold's action sequence as the overall logic, this study explores the connections between abusive supervision and job performance. Our findings suggest that abusive supervision has a significant positive impact on job performance. Furthermore, challenge stressors and innovative work behavior partially mediate the relationship between abusive supervision and job performance, and these two factors also form a chain mediating effect. Leader-member exchange moderates the relationship between abusive supervision and job performance, as higher levels of leader-member exchange are associated with a stronger positive effect of abusive supervision on job performance.

\section{Acknowledgment}

This research was supported by Hunan Key Laboratory of Macroeconomic Big Data Mining and its Application; Hunan Provincial Social Science Achievement Review Committee Project (No. XSP22YBC199); The Outstanding Young Project of Education Department of Hunan Province (No. 21B0051).

\section{Disclosure}

The authors report no conflicts of interest in this work. 


\section{References}

1. Tepper BJ. Consequences of abusive supervision. Acad Manage J. 2000;43(2):178-190. doi:10.5465/1556375

2. Tepper BJ. Abusive supervision in work organizations: review, synthesis, and research agenda. J Manage. 2007;33(3):261-289. doi:10.1177/ 0149206307300812

3. Islam T, Ahmad S, Kaleem A, Mahmood K. Abusive supervision and knowledge sharing: moderating roles of Islamic work ethic and learning goal orientation. Manage Decis. 2020;59(2):205-222. doi:10.1108/MD-08-2019-1069

4. Oh JK, Farh C. An emotional process theory of how subordinates appraise, experience, and respond to abusive supervision over time. Acad Manage Rev. 2017;42(2):207-232. doi:10.5465/amr.2014.0347

5. Liao Z, Peng AC, Li W, Schaubroeck J. Is abuse always bad? A latent change score approach to examine consequences of abusive supervision. Acad Manag Proc. 2016;2016(1):11621. doi:10.5465/ambpp.2016.235

6. Wang R, Jiang J. How abusive supervisors influence employees' voice and silence: the effects of interactional justice and organizational attribution. J Soc Psychol. 2016;155(3):204-220. doi:10.1080/00224545.2014.990410

7. Lee S, Yun S, Srivastava A. Evidence for a curvilinear relationship between abusive supervision and creativity in South Korea. Leadersh $Q$. 2013;24(5):724-731. doi:10.1016/j.leaqua.2013.07.002

8. Islam T, Asad M. Enhancing employees' creativity through entrepreneurial leadership: can knowledge sharing and creative self-efficacy matter? VINE J Inf Knowl Manag Syst. 2021; ahead-of-print(ahead-of-print). doi:10.1108/VJIKMS-07-2021-0121

9. Zhang J, Liu J. Is abusive supervision an absolute devil? Literature review and research agenda. Asia Pac J Manag. 2018;35(3):719-744. doi:10.1007/s10490-017-9551-y

10. Bakker AB, Demerouti E. The job demands-resources model: state of the art. J Manage Psychol. 2007;22(3):309-328. doi:10.1108/ 02683940710733115

11. Crawford ER, LePine JA, Rich BL. Linking job demands and resources to employee engagement and burnout: a theoretical extension and meta-analytic test. J Appl Psychol. 2010;95(5):834-848. doi:10.1037/a0019364

12. Iqbal Q, Ahmad NH, Ahmad B. Enhancing sustainable performance through job characteristics via workplace spirituality: a study on SMEs. J Sci Technol Policy Manag. 2021;12(3):463-490. doi:10.1108/JSTPM-02-2018-0022

13. Iqbal Q, Ahmad NH, Nasim A, Khan SAR. A moderated-mediation analysis of psychological empowerment: sustainable leadership and sustainable performance. J Clean Prod. 2020;262:121429. doi:10.1016/j.jclepro.2020.121429

14. Cavanaugh MA, Boswell WR, Roehling MV, Boudreau JW. An empirical examination of self-reported work stress among U.S. managers. $J$ Appl Psychol. 2000;85(1):65-74. doi:10.1037/0021-9010.85.1.65

15. Scott SG, Bruce RA. Determinants of innovative behavior: a path model of individual innovation in the workplace. Acad Manage J. 1994;37 (3):580-607. doi:10.5465/256701

16. Gasper K, Bramesfeld KD. Imparting wisdom: Magda Arnold's contribution to research on emotion and motivation. Cognition Emotion. 2006;20 (7):1001-1026. doi:10.1080/02699930600616122

17. Bakker AB, Demerouti E, Taris TW, Schaufeli WB, Schreurs PJG. A multigroup analysis of the job demands-resources model in four home care organizations. Int J Stress Manage. 2003;10(1):16-38. doi:10.1037/1072-5245.10.1.16

18. Yu L, Duffy MK. The whiplash effect: the (moderating) role of attributed motives in emotional and behavioral reactions to abusive supervision. J Appl Psychol. 2021;106(5):754-773. doi:10.1037/apl0000810

19. Fan Y. Guanxi's consequences: personal gains at social cost. J Bus Ethics. 2002;38(4):371-380. doi:10.2307/25074806

20. Graen GB, Uhl-Bien M. Relationship-based approach to leadership: development of leader-member exchange (LMX) theory of leadership over 25 years: applying a multi-level multi-domain perspective. Leadersh Q. 1995;6(2):219-247. doi:10.1016/1048-9843(95)90036-5

21. Jiang JY, Law KS, Sun AJJM. Leader - member relationship and burnout: the moderating role of leader integrity. Manage Organ Rev. 2014;10 (2):223-247. doi:10.1111/more.12022

22. Chen X, Eberly MB, Chiang T, Farh J, Cheng B. Affective trust in Chinese leaders: linking paternalistic leadership to employee performance. J Manage. 2014;40(3):796-819. doi:10.1177/0149206311410604

23. Pellegrini EK, Scandura TA. Paternalistic leadership: a review and agenda for future research. J Manage. 2008;34(3):566-593. doi:10.1177/ 0149206308316063

24. Kim KY, Atwater L, Latheef Z, Zheng D. Three motives for abusive supervision: the mitigating effect of subordinates attributed motives on abusive supervision's negative outcomes. J Leadersh Org Stud. 2018;26(4):476-494. doi:10.1177/1548051818781816

25. Xu AJ, Loi R, Lam LW. The bad boss takes it all: how abusive supervision and leader-member exchange interact to influence employee silence. Leadersh Q. 2015;26(5):763-774. doi:10.1016/j.leaqua.2015.03.002

26. Shao P, Li A, Mawritz M. Self-protective reactions to peer abusive supervision: the moderating role of prevention focus and the mediating role of performance instrumentality. J Organ Behav. 2018;39(1):12-25. doi:10.1002/job.2206

27. Thoroughgood CN, Tate BW, Sawyer KB, Jacobs R. Bad to the Bone. J Leadersh Org Stud. 2012;19(2):230-255. doi:10.1177/1548051811436327

28. Liu D, Liao H, Loi R. The dark side of leadership: a three-level investigation of the cascading effect of abusive supervision on employee creativity. Acad Manage J. 2012;55(5):1187-1212. doi:10.5465/amj.2010.0400

29. Chan ME, McAllister DJ. Abusive supervision through the lens of employee state paranoia. Acad Manage Rev. 2014;39(1):44-66. doi:10.5465/ amr.2011.0419

30. Treadway DC, Yang J, Bentley JR, Williams LV, Reeves M. The impact of follower narcissism and LMX perceptions on feeling envied and job performance. Int J Hum Resour Man. 2019;30(7):1181-1202. doi:10.1080/09585192.2017.1288151

31. LePine JA, LePine MA, Jackson CL. Challenge and hindrance stress: relationships with exhaustion, motivation to learn, and learning performance. J Appl Psychol. 2004;89(5):883-891. doi:10.1037/0021-9010.89.5.883

32. Halbesleben JRB, Neveu J, Paustian-Underdahl SC, Westman M. Getting to the "COR": understanding the role of resources in conservation of resources theory. J Manage. 2014;40(5):1334-1364. doi:10.1177/0149206314527130

33. Boswell WR, Olson-Buchanan JB, LePine MA. Relations between stress and work outcomes: the role of felt challenge, job control, and psychological strain. J Vocat Behav. 2004;64(1):165-181. doi:10.1016/S0001-8791(03)00049-6 
34. Baumeister RF, Bratslavsky E, Finkenauer C, Vohs KD. Bad is stronger than good. Rev Gen Psychol. 2001;5(4):323-370. doi:10.1037//10892680.5.4.323

35. Lyu Y, Zhou X, Li W, et al. The impact of abusive supervision on service employees' proactive customer service performance in the hotel industry. Int J Contemp Hosp M. 2016;28(9):1992-2012. doi:10.1108/ijchm-03-2015-0128

36. Amabile TM, Hadley CN, Kramer SJ. Creativity under the gun. Harvard Bus Rev. 2002;80(8):52-61, 147. doi:10.1016/S0957-4174(02)00034-9

37. Tsui AS, Farh J. Where guanxi matters. Work Occup. 1997;24(1):56-79. doi:10.1177/0730888497024001005

38. Afsar B, Badir Y, Khan MM. Person-job fit, person-organization fit and innovative work behavior: the mediating role of innovation trust. $J$ High Technol Manag Res. 2015;26(2):105-116. doi:10.1016/j.hitech.2015.09.001

39. Sacramento CA, Fay D, West MA. Workplace duties or opportunities? Challenge stressors, regulatory focus, and creativity. Organ Behav Hum Dec. 2013;121(2):141-157. doi:10.1016/j.obhdp.2013.01.008

40. Sonnentag S, Spychala A. Job control and job stressors as predictors of proactive work behavior: is role breadth self-efficacy the link? Hum Perform. 2012;25(5):412-431. doi:10.1080/08959285.2012.721830

41. Byron K, Khazanchi S, Nazarian D. The relationship between stressors and creativity: a meta-analysis examining competing theoretical models. J Appl Psychol. 2010;95(1):201-212. doi:10.1037/a0017868

42. Zhang Y, LePine JA, Buckman BR, Wei F. It's not fair ... or is it? The role of justice and leadership in explaining work stressor-job performance relationships. Acad Manage J. 2014;57(3):675-697. doi:10.5465/amj.2011.1110

43. Bakker AB, Hakanen JJ, Demerouti E, Xanthopoulou D. Job resources boost work engagement, particularly when job demands are high. $J$ Educ Psychol. 2007;99(2):274-284. doi:10.1037/0022-0663.99.2.274

44. Mischel W, Shoda Y. A cognitive-affective system theory of personality: reconceptualizing situations, dispositions, dynamics, and invariance in personality structure. Psychol Rev. 1995;102(2):246-268. doi:10.1037/0033-295x.102.2.246

45. Ilies R, Nahrgang JD, Morgeson FP. Leader-member exchange and citizenship behaviors: a meta-analysis. J Appl Psychol. 2007 ;92(1):269-277. doi:10.1037/0021-9010.92.1.269

46. Lian H, Lance Ferris D, Brown DJ. Does taking the good with the bad make things worse? How abusive supervision and leader-member exchange interact to impact need satisfaction and organizational deviance. Organ Behav Hum Dec. 2012;117(1):41-52. doi:10.1016/j. obhdp.2011.10.003

47. Aryee S, Sun L, Chen ZXG, Debrah YA. Abusive supervision and contextual performance: the mediating role of emotional exhaustion and the moderating role of work unit structure. Manage Organ Rev. 2008;4(3):393-411. doi:10.1111/j.1740-8784.2008.00118.x

48. Chen ZX, Tsui AS, Farh J. Loyalty to supervisor vs. organizational commitment: relationships to employee performance in China. J Occup Organ Psych. 2002;75(3):339-356. doi:10.1348/096317902320369749

49. Podsakoff PM, MacKenzie SB, Lee J, Podsakoff NP. Common method biases in behavioral research: a critical review of the literature and recommended remedies. J Appl Psychol. 2003;88(5):879-903. doi:10.1037/0021-9010.88.5.879

50. Petter S, Straub D, Rai A. Specifying formative constructs in information systems research. Mis Quart. 2007;31(4):623-656. doi:10.2307/25148814

51. Chen S, Lin C. Understanding the effect of social media marketing activities: the mediation of social identification, perceived value, and satisfaction. Technol Forecast Soc. 2019;140:22-32. doi:10.1016/j.techfore.2018.11.025

52. Fornell C, Larcker DF. Evaluating structural equation models with unobservable variables and measurement error. $J$ Marketing Res. 1981;18 (1):39-50. doi:10.1177/002224378101800104

53. Chin WW. The partial least squares approach to structural equation modeling. In Modern Methods for Business Research; 1998 : $295-336$. Available from: https://www.researchgate.net/publication/311766005. Accessed February 16, 2022.

54. Thatcher JB, Perrewe PL. An empirical examination of individual traits as antecedents to computer anxiety and computer self-efficacy. Mis Quart. 2002;26(4):381-396. doi:10.2307/4132314

55. Henseler J, Ringle CM, Sinkovics RR. The use of partial least squares path modeling in international marketing. Adv Int Mark. 2009;20:277-320. doi:10.1108/S1474-7979(2009)0000020014

56. Baer MD, Dhensa-Kahlon RK, Colquitt JA, et al. Uneasy lies the head that bears the trust: the effects of feeling trusted on emotional exhaustion. Acad Manage J. 2015;58(6):1637-1657. doi:10.5465/amj.2014.0246

Psychology Research and Behavior Management

Dovepress

Publish your work in this journal

Psychology Research and Behavior Management is an international, peer-reviewed, open access journal focusing on the science of psychology and its application in behavior management to develop improved outcomes in the clinical, educational, sports and business arenas. Specific topics covered in the journal include: Neuroscience, memory and decision making; Behavior modification and management; Clinical applications; Business and sports performance management; Social and developmental studies; Animal studies. The manuscript management system is completely online and includes a very quick and fair peer-review system, which is all easy to use. Visit http://www.dovepress.com/testimonials.php to read real quotes from published authors.

Submit your manuscript here: https://www.dovepress.com/psychology-research-and-behavior-management-journal 